VoL. 43 (1991) [169-175]

\title{
THE SMOOTH VARIATIONAL PRINCIPLE AND GENERIC DIFFERENTIABILITY
}

\author{
Pando Grigorov Grorgiev
}

\begin{abstract}
A modified version of the smooth variational principle of Borwein and Preiss is proved. By its help it is shown that in a Banach space with uniformly Gateaux differentiable norm every continuous function, which is directionally differentiable on a dense $G_{\delta}$ subset of the space, is Gâteaux differentiable on a dense $G_{\delta}$ subset of the space.
\end{abstract}

The question of generic differentiability (that is, on a dense $G_{\delta}$ subset of the domain) of non-convex functions has been considered by many authors. First results in this direction were obtained by Kenderov in [7] where he proved that in a separable Banach space a continuous and quasi-differentiable in the sense of Pshenichniy function as well as a locally Lipschitz and directionally differentiable function is generic Gâteaux differentiable. Later, Lau and Weil [9], Fabian [4], Lebourg [10] have obtained generalisations of Kenderov's results in several directions, but only in the separable case. In the non-separable case analogous extensions are proved by Zhivkov [13, 14]. Other types of results about generic Frechet differentiability of non-convex functions are obtained by Ekeland and Lebourg [3], Zajicek [12], Fabian [4], de Barra, Fitzpatrick and Giles [1], Georgiev [5], et cetera.

In this paper, by a modification of the smooth variational principle of Borwein and Preiss [2], we establish a result stating that in a Banach space with uniformly Gâteaux differentiable norm, every continuous, directionally differentiable on a dense $G_{\delta}$ subset of its domain, function is generic Gâteaux differentiable.

Let $E$ be a Banach space. The function $f: E \rightarrow \mathbf{R}$ is said to be directionally differentiable at $x_{0}$ if for every $h \in E$ the one-sided directional derivative $f^{\prime}\left(x_{0} ; h\right)=$ $\lim _{t \downarrow 0}\left(f\left(x_{0}+t h\right)-f\left(x_{0}\right)\right) / t$ exists. The function $f$ is Gâteaux differentiable at $x_{0}$ if the operator $f^{\prime}\left(x_{0} ;.\right)$ is continuous and linear. In this case $f^{\prime}\left(x_{0} ;.\right)$ is denoted by $\nabla f\left(x_{0}\right)$.

The following assertion is a slight modification of the Borwein-Preiss smooth variational principle [2] and is essential in the sequel.

Received 22 March 1990

Copyright Clearance Centre, Inc. Serial-fee code: 0004-9729/91 \$A2.00+0.00. 
Theorem 1. Let $E$ be a Banach space, $X \subset E$ be a closed non-empty subset, $f: X \rightarrow R \cup\{+\infty\}$ be a lower semicontinuous function and $\varepsilon>0, \lambda>0, p \geqslant 1$ be given. Suppose that $x_{0}$ satisfies the condition

$$
\inf _{x \in X}\left\{f(x)+\frac{\varepsilon}{\lambda^{p}}\left\|x-x_{0}\right\|^{p}\right\}<f\left(x_{0}\right)<\inf f(X)+\varepsilon .
$$

Then there exists $r_{1}>0$ such that for every $r_{2}>0$ there exist a point $v \in X$, a sequence $\left\{x_{n}\right\}_{n=0}^{\infty} \subset X$ converging to $v$, s sequence $\left\{\mu_{n}\right\}_{n=0}^{\infty} \subset[0,1]$ with $\sum_{n=0}^{\infty} \mu_{n}=1$ such that

(1) $f(v)+\left(\varepsilon / \lambda^{p}\right) \Delta(v) \leqslant f(x)+\left(\varepsilon / \lambda^{p}\right) \Delta(x) \forall x \in X$, where

(2) $\Delta(x)=\sum_{n=0}^{\infty} \mu_{n}\left\|x-x_{n}\right\|^{p}$,

(3) $\left\|v-x_{0}\right\|<\lambda$,

(4) $\left\|x_{n}-v\right\|<r_{2} \forall n \geqslant 1$,

(5) $\left\|x_{1}-x_{0}\right\| \geqslant r_{1}$

Proof: Choose $\varepsilon^{\prime}<\varepsilon$ such that $f\left(x_{0}\right)<\inf f(X)+\varepsilon^{\prime}$ and put $\mu_{n}=\left(1-q_{1}\right) q_{1}^{n}$, $n=0,1,2 \ldots$, where $q_{1} \in\left(0, \min \left\{1,\left(\varepsilon-\varepsilon^{\prime}\right) / \varepsilon\right\}\right)$. For fixed $\delta \in\left(0, f\left(x_{0}\right)-\inf _{x \in X}\left\{f(x)+\mu_{0} \varepsilon / \lambda^{P}\left\|x-x_{0}\right\|^{p}\right\}\right)$ denote

$$
X_{\delta}=\left\{x \in X: f(x)+\mu_{0} \frac{\varepsilon}{\lambda^{p}}\left\|x-x_{0}\right\|^{p} \leqslant \inf _{z \in X}\left\{f(z)+\mu_{0} \frac{\varepsilon}{\lambda^{p}}\left\|z-x_{0}\right\|^{p}\right\}+\delta\right\} .
$$

The set $X_{\delta}$ is closed (because $f$ is lower semicontinuous) and since $x_{0} \notin X_{\delta}$ we have $r_{1}:=\operatorname{dist}\left(x_{0}, X_{\delta}\right):=\inf _{x \in X_{\delta}}\left\|x_{0}-x\right\|>0$.

Let $r_{2}>0$ be fixed. Choose $q_{2} \in\left(0, \min \left\{q_{1}, \delta / \varepsilon^{\prime}\right\}\right)$ such that for $q:=\left(q_{2} / q_{1}\right)^{1 / p}$ to be fulfilled:

$$
s:=\left(\frac{1+q_{2}}{1-q_{1}}\right)^{1 / p} \cdot \frac{1}{1-q} \cdot\left(\frac{\varepsilon^{\prime}}{\varepsilon}\right)^{1 / p}<1 \text { and } \lambda s q<r_{2} .
$$

Define inductively the functions $\left\{f_{n}\right\}_{n=0}^{\infty}$ and the points $\left\{x_{n}\right\}_{n=0}^{\infty}$ by:

$$
f_{n+1}(x)=f_{n}(x)+\frac{\varepsilon}{\lambda^{p}} \mu_{n}\left\|x-x_{n}\right\|^{p}, \quad f_{0}:=f ;
$$

$x_{n}$ is such that

$$
f_{n}\left(x_{n}\right)<\inf f_{n}(X)+\varepsilon_{n} \text { where } \varepsilon_{n}=\varepsilon^{\prime} q_{2}^{n}, n=0,1,2, \ldots
$$

Since $x_{1} \in X_{\delta}$, we have $\left\|x_{0}-x_{1}\right\| \geqslant \operatorname{dist}\left(x_{0}, X_{\delta}\right)=r_{1}$, which is (5). 
Using (7) and (8) we can write

$$
\begin{aligned}
& \mu_{n} \frac{\varepsilon}{\lambda^{p}}\left\|x_{n+1}-x_{n}\right\|^{p}=f_{n+1}\left(x_{n+1}\right)-f_{n}\left(x_{n+1}\right) \\
& =f_{n+1}\left(x_{n+1}\right)-f_{n+1}\left(x_{n}\right)+f_{n}\left(x_{n}\right)-f_{n}\left(x_{n+1}\right)<\varepsilon_{n+1}+\varepsilon_{n} .
\end{aligned}
$$

Hence

$$
\begin{aligned}
\left\|x_{n+1}-x_{n}\right\|<\lambda\left(\frac{\varepsilon_{n+1}+\varepsilon_{n}}{\varepsilon \mu_{n}}\right)^{1 / p} & =\lambda\left(\frac{q_{2}^{n}\left(q_{2}+1\right)}{q_{1}^{n}\left(1-q_{1}\right)}\right)^{1 / p}\left(\frac{\varepsilon^{\prime}}{\varepsilon}\right)^{1 / p} \\
& =\lambda q^{n}\left(\frac{q_{2}+1}{1-q_{1}}\right)^{1 / p}\left(\frac{\varepsilon^{\prime}}{\varepsilon}\right)^{1 / p}
\end{aligned}
$$

and having in mind the notion in (6), for $m>n$ we obtain

$$
\left\|x_{m}-x_{n}\right\|<\lambda s\left(1-q^{m-n}\right) q^{n} .
$$

This shows that $\left\{x_{n}\right\}_{n=0}^{\infty}$ is a fundamental sequence, therefore there exists a point $v$ such that $x_{n} \rightarrow v$. Now assertions (3) and (4) follow by (9).

To establish (1), let $\gamma>0$ be given. Since $f$ is lower semicontinuous and $\Delta$ (defined by (2)) is continuous, there exists $\delta>0$ such that

$$
f(v)+\frac{\varepsilon}{\lambda^{p}} \Delta(v)<f(x)+\frac{\varepsilon}{\lambda^{p}} \Delta(x)+\gamma / 3 \text { whenever }\|x-v\|<\delta .
$$

Choose $n$ sufficiently large such that $\varepsilon_{n}<\gamma / 3,\left\|x_{n}-v\right\|<\delta$ and $\left(\varepsilon / \lambda^{p}\right) \sum_{k=n}^{\infty} \mu_{k}\left\|x_{n}-x_{k}\right\|^{p}<\gamma / 3$. For every $x \in X$, using (10), (7) and (8), we can write

$$
\begin{aligned}
f(v)+\frac{\varepsilon}{\lambda^{p}} \Delta(v) & <f\left(x_{n}\right)+\frac{\varepsilon}{\lambda^{p}} \Delta\left(x_{n}\right)+\gamma / 3 \\
& =f_{n}\left(x_{n}\right)+\frac{\varepsilon}{\lambda^{p}} \sum_{k=n}^{\infty} \mu_{k}\left\|x_{n}-x_{k}\right\|^{p}+\gamma / 3 \\
& <f_{n}(x)+\varepsilon_{n}+\gamma / 3+\gamma / 3<f(x)+\frac{\varepsilon}{\lambda^{p}} \Delta(x)+\gamma
\end{aligned}
$$

and (1) is proved.

ThEOREM 2. Let the Banach space $E$ have a uniformly Gâteaux differentiable norm (this means that the norm is Gâteaux differentiable on $E \backslash\{0\}$ and the limit $\lim _{t ! 0}(\|x+t h\|-\|x\|) / t$ is uniform with respect to $\left.x \in S:=\{x \in E:\|x\|=1\}\right)$. Then every continuous function defined on an open subset $D \subset E$, which is directionally 
differentiable on a dense $G_{\delta}$ subset of $D$, is Gâteaux differentiable on a dense $G_{\delta}$ subset of $D$.

Proof: By Proposition 2.1 of [14], $f$ is locally Lipschitzian on a dense and open subset $D_{1}$ of $D$. Let $U \subset D_{1}$ be an open subset such that $f$ is Lipschitz on $U$. If we prove that $f$ is Gateaux differentiable on a dense $G_{\delta}$ subset of $U$, then the theorem would be proved, having in mind the localisation principle (see [8], Chapter I, Section 10, V) stating that a subset $P$ of a topological space is of first Baire category if for every point $p \in P$ there exists an open set $H \ni p$ such that $P \cap H$ is of first Baire category in $H$.

Define the sets:

$$
\begin{aligned}
& X_{n}^{\prime}=\left\{x \in U: \exists p_{n} \in(1,2), \quad \exists t_{n} \in\left(0,\left(\frac{1}{n}\right)^{\frac{1}{p_{n}-1}}\right), \quad \exists\left\{x_{n, m}\right\}_{m=0}^{\infty} \subset U,\right. \\
& \exists\left\{\mu_{n, m}\right\}_{m=0}^{\infty} \subset[0,1], \sum_{m=0}^{\infty} \mu_{n, m}=1, \exists x_{n} \subset U: x_{n, m} \rightarrow x_{n},\left\|x-x_{n}\right\|<t_{n}^{2}, \\
& B\left(x_{n} ; 2 t_{n}\right) \subset U,\left\|x_{n, m}-x_{n}\right\|<t_{n}^{2} \forall m \geqslant 1,2 t_{n}<\left\|x_{n, 0}-x_{n, 1}\right\|^{2}<1 / n^{2} \text { and } \\
& f(x)+2 \triangle_{n}(x)<\inf _{x \in B\left(x_{n} ; 2 t_{n}\right)}\left\{f(z)+2 \triangle_{n}(z)\right\}+t_{n}^{2}, \text { where } \\
& \left.\triangle_{n}(y)=\sum_{m=0}^{\infty} \mu_{n, m}\left\|y-x_{n, m}\right\|^{p_{n}}\right\}, \\
& X_{n}^{\prime \prime}=\left\{x \in U: \exists p_{n} \in(1,2), \exists t_{n} \in\left(0,\left(\frac{1}{n}\right)^{\frac{1}{p_{n}-1}}\right), \exists x_{n} \subset U:\left\|x-x_{n}\right\|<t_{n}^{2},\right. \\
& \left.B\left(x_{n} ; 2 t_{n}\right) \subset U, f(x)<\underset{z \in B\left(x_{n} ; 2 t_{n}\right)}{\inf _{x}}\left\{f(z)+2\|x-z\|^{p_{n}}\right\}+t_{n}^{2}\right\} .
\end{aligned}
$$

Since $f$ is continuous, the sets $X_{n}^{\prime}$ and $X_{n}^{\prime \prime}$ are open. We shall prove that the set $X_{n}:=X_{n}^{\prime} \cup X_{n}^{\prime \prime}$ is dense in $U$.

Let $n \geqslant 2, x_{n, 0} \in U$ be fixed. Choose $\varepsilon \in(0,1 / n)$ in such a way that $B\left[x_{n, 0} ; \varepsilon\right] \subset$ $U$. For $p_{n} \in(1,(\ln \varepsilon / 2) /(\ln \varepsilon))$ we put $\lambda=(\varepsilon / 2)^{1 / p_{n}}$. So we have $\lambda<2 \lambda^{p_{n}}=\varepsilon$. Having in mind that if $\alpha f$ for some $\alpha>0$ is Gateaux differentiable at a point $x$ then $f$ is also Gâteaux differentiable at $x$, we may assume without loss of generality that the Lipschitz constant of $f$ is less than 1 . We can write

$$
f\left(x_{n, 0}\right) \leqslant \inf _{z \in B\left[x_{n, 0} ; \varepsilon\right]}\left\{f(z)+L\left\|x_{n, 0}-z\right\|\right\}<\inf _{z \in B\left[x_{n, 0 ; \varepsilon]}\right.} f(z)+\varepsilon .
$$

If $f\left(x_{n, 0}\right) \leqslant f(x)+2\left\|x-x_{n, 0}\right\|^{p_{n}}$ for every $x \in B\left[x_{n, 0} ; \varepsilon\right]$, then $x_{n, 0} \in X_{n}^{\prime \prime}$ for $t_{n} \in$ $\left(0, \min \left\{\varepsilon / 2,(1 / n)^{1 /\left(p_{n}-1\right)}\right\}\right)$. If this is not true, we apply Theorem 1 with $\lambda, \varepsilon$, 
$p_{n}$ defined above and $X=B\left[x_{n, 0} ; \varepsilon\right]$. So there exists $r_{1}>0$ such that for $r_{2}=t_{n}^{2}$ where $t_{n} \in\left(0, \min \left\{(\varepsilon-\lambda) / 2,(1 / n)^{1 /\left(p_{n}-1\right)}, r_{1}^{2} / 2\right\}\right)$ we obtain a point $x_{n}$, sequence $\left\{x_{n, m}\right\}_{m=0}^{\infty} \subset X, x_{n, m} \rightarrow x_{n},\left\{\mu_{n, m}\right\}_{m=0}^{\infty} \subset[0,1], \sum_{m=0}^{\infty} \mu_{n, m}=1$ with the following properties:

where

$$
f\left(x_{n}\right)+2 \triangle_{n}\left(x_{n}\right)=\inf _{z \in X}\left\{f(z)+2 \triangle_{n}(z)\right\}
$$

$$
\begin{aligned}
& \triangle_{n}(y)=\sum_{m=0}^{\infty} \mu_{n, m}\left\|y-x_{n, m}\right\|^{p_{n}}, \\
& \left\|x_{n}-x_{n, 0}\right\|<\lambda<2 \lambda^{p}=\varepsilon,
\end{aligned}
$$$$
\left\|x_{n, m}-x_{n}\right\|<t_{n}^{2} \quad \forall m \geqslant 1 \text {, }
$$$$
\left\|x_{n, 0}-x_{n, 1}\right\| \geqslant r_{1}>\left(2 t_{n}\right)^{1 / 2} \text {. }
$$

Regarding the proof of Theorem 1 we can see that $\left\|x_{n, 0}-x_{n, 1}\right\|<\lambda<\varepsilon<1 / n$. Also by the choice of $t_{n}$ and by (12) we have $B\left(x_{n} ; 2 t_{n}\right) \subset X$. Now by (11), (12), (13) and (14) the denseness is proved.

By the Baire category theorem the set $X_{0}=\bigcap_{n=2}^{\infty} X_{n}$ is dense and $G_{\delta}$ in $U$. We shall prove that $f$ is Gâteaux differentiable on $X_{0}$. Let $x_{0} \in X_{0}$. Consider the cases:

CASE 1. $x_{0}$ belongs to infinitely many $X_{n}$. Without loss of generality, we can assume that $x_{0} \in X_{n}$ for every $n \geqslant 2$. Let $p_{n}, t_{n}, x_{n},\left\{x_{n, m}\right\}_{m=0}^{\infty},\left\{\mu_{n, m}\right\}_{m=0}^{\infty}$ be the elements from the definition of $X_{n}^{\prime}$ corresponding to $x_{0}$. It is easy to check that $x_{0} \neq x_{n, 0}$ for $n \geqslant 2$. Let $t_{n}^{\prime}=t_{n} /\left\|x_{0}-x_{n, 0}\right\|$ and $z_{n}=\left(x_{0}-x_{n, 0}\right) /\left\|x_{0}-x_{n, 0}\right\|$. Since

$$
\begin{gathered}
\left\|x_{0}-x_{n, 1}\right\| \leqslant\left\|x_{0}-x_{n}\right\|+\left\|x_{n}-x_{n, 1}\right\|<2 t_{n}^{2} \\
\quad<\left\|x_{n, 1}-x_{n, 0}\right\|^{4} / 2<\left\|x_{n, 1}-x_{n, 0}\right\| / 2
\end{gathered}
$$

we have

$$
t_{n}^{\prime} \leqslant \frac{t_{n}}{\left\|x_{n, 1}-x_{n, 0}\right\|-\left\|x_{n, 1}-x_{0}\right\|}<\frac{2 t_{n}}{\left\|x_{n, 1}-x_{n, 0}\right\|}<\left\|x_{n, 1}-x_{n, 0}\right\|<1 / n .
$$

Since $\|\cdot\|^{p}, p \geqslant 1$, is a convex function, it is locally Lipschitz. From the proof of this fact (see for instance [11], p.4) we can see that the functions $\|\cdot\|^{p}, p \in(1,2)$ are Lipschitz on the unit ball with one and the same Lipschitz constant $L$. Without loss of generality we may assume that $\nabla\left\|z_{n}\right\| \stackrel{\omega^{*}}{\longrightarrow} z_{0}^{*}$, where $\nabla\|z\|$ denotes the Gâteaux derivative of the norm at $z$ (choosing a convergent subsequence if it is necessary) because the closed dual unit ball is sequentially $\omega^{*}$-compact (see $[6]$ ) and $\|\nabla\| z_{n}\|\|^{*}=1$. 
Since the norm is uniformly Gâteaux differentiable, it is a routine matter to prove that for every $\varepsilon>0$ and $h \in S$ there exists $\delta>0$ such that

$$
\frac{\|x+t h\|^{p}-\|x\|^{p}}{t}-p\langle\nabla\|x\|, h\rangle<\varepsilon \quad \forall x \in S, \forall t \in(0, \delta), \forall p \in[1,2] .
$$

For every $\varepsilon>0$ and such $\delta$, for $n>1 / \delta, h \in S$, since $x_{0}+t_{n} h \in B\left(x_{n} ; 2 t_{n}\right)$, using (15), we can write

$$
\begin{aligned}
& \frac{f\left(x_{0}+t_{n} h\right)-f\left(x_{0}\right)}{t_{n}} \geqslant-\frac{2 \triangle_{n}\left(x_{0}+t_{n} h\right)-2 \triangle_{n}\left(x_{0}\right)}{t_{n}}-t_{n} \\
& =-2 \sum_{m=0}^{\infty} \mu_{n, m} \frac{\left\|x_{0}+t_{n} h-x_{n, m}\right\|^{p_{n}}-\left\|x_{0}-x_{n, m}\right\|^{p_{n}}}{t_{n}}-t_{n} \\
& \geqslant-2 \mu_{n, 0} \frac{\left\|x_{0}+t_{n} h-x_{n, 0}\right\|^{p_{n}}-\left\|x_{0}-x_{n, 0}\right\|^{p_{n}}}{t_{n}} \\
& \quad-2 \sum_{m=1}^{\infty} \mu_{n, m} \frac{\left\|t_{n} h\right\|^{p_{n}}+L\left\|x_{0}-x_{n, m}\right\|}{t_{n}}-t_{n} \\
& \geqslant-2 \mu_{n, 0} \frac{\left\|z_{n}+t_{n}^{\prime} h\right\|^{p_{n}}-\left\|z_{n}\right\|^{p_{n}}}{t_{n}^{\prime}}\left\|x_{0}-x_{n, 0}\right\|^{p_{n}-1}-2 t_{n}^{p_{n}-1}-4 L t_{n}-t_{n} \\
& >-2 p_{n}\left\langle\nabla\left\|z_{n}\right\|, h\right\rangle-2 \varepsilon-2 / n-4 L / n-1 / n \\
& >-4\left\langle\nabla\left\|z_{n}\right\|, h\right\rangle-2 \varepsilon-3 / n-4 L / n .
\end{aligned}
$$

Hence, for $z_{1}^{*}=-4 z_{0}^{*}$, after passing to limits, we obtain

$$
f^{\prime}\left(x_{0} ; h\right) \geqslant\left\langle z_{1}^{*}, h\right\rangle-2 \varepsilon
$$

and since this is valid for every $\varepsilon>0$ and $h \in S$, we have

$$
f^{\prime}\left(x_{0} ; h\right) \geqslant\left\langle z_{1}^{*}, h\right\rangle \quad \forall h \in S .
$$

CASE 2. If Case 1 is not fulfilled, then $x_{0}$ belongs to infinitely many $X_{n}^{\prime \prime}$. Without loss of generality we may assume that $x_{0} \in X_{n}^{\prime \prime}$ for every $n \geqslant 1$. Let $t_{n}, p_{n}$ and $x_{0}$ be the elements from the definition of $X_{n}^{\prime \prime}$ corresponding to $x_{0}$. We can write

$$
\frac{f\left(x_{0}+t_{n} h\right)-f\left(x_{0}\right)}{t_{n}} \geqslant-t_{n}^{p_{n}-1}-t_{n}>-2 / n .
$$

Hence, after passing to limits, we get $f^{\prime}\left(x_{0} ; h\right) \geqslant 0$.

Repeating this reasoning for the function $-f$, we obtain a dense $G_{\delta}$ subset in $U$ at every point $x$ of which it is fullfilled: there exist $x_{1}^{*}, x_{2}^{*} \in E^{*}$ such that $\left\langle x_{2}^{*}, h\right\rangle \geqslant f^{\prime}(x ; h) \geqslant\left\langle x_{1}^{*}, h\right\rangle$ for every $h \in S$. Hence $x_{1}^{*}=x_{2}^{*}=\nabla f(x)$ and the proof is completed.

The proof of Theorem 2 shows that a Banach space with uniformly Gâteaux differentiable norm is a $\lambda$-space in the terminology of [14]. 


\title{
REFERENCES
}

[1] G. de Barra, S. Fitzpatrick and J.R. Giles, 'On generic differentiability of locally Lipschitz functions on Banach space', (preprint).

[2] J. Borwein and D. Preiss, 'A smooth variational principle with applications to subdifferentiability and to differentiability of convex functions', Trans. Amer. Math. Soc. 303 (1987), 517-527.

[3] I. Ekeland and G. Lebourg, 'Generic Frechet differentiability and perturbed optimization problems in Banach spaces', Trans. Amer. Math. Soc. 224 (1976), 193-216.

[4] M. Fabian, 'Differentiability via one sided directional derivatives', Proc. Amer. Math. Soc. 82 (1982), 495-500.

[5] P. Gr. Georgiev, 'Locally Lipschitz and regular functions are Frechet differentiable almost every where in Asplund spaces', Comp. Rend. Acad. Bulg. Sci. 42 (1989), 13-15.

[6] J. Hagler and F. Sullivan, 'Smoothness and weak* sequential compactness', Proc. Amer. Math. Soc. 78 (1980), 497-503.

[7] P.S. Kenderov, 'The quasi-differentiable functionals are almost every where differentiable', in Math. and Education Math., Proc. 2nd Spring Conf. Bulg. Math. Soc. pp. 123-126 (Vidin, 1973).

[8] K. Kuratowski, Topology I (Academic Press, New York and London, 1966).

[9] Ka-Sing Lau and C.E. Weil, 'Differentiability via directional derivatives', Proc. Amer. Math. Soc. 70 (1978), 11-17.

[10] G. Lebourg, 'Generic differentiability of Lipschitzian functions', Trans. Amer. Math. Soc. 256 (1979), 125-144.

[11] R.R. Phelps, 'Convex functions, monotone operators and differentiability', in Lecture Notes in Math 1364, 1989.

[12] L. Zajicek, 'A generalization of an Ekeland-Lebourg theorem and the differentiability of distance functions', Rend. Circ. Mat. Palermo Suppl 3 (1984), 403-410.

[13] N.V. Zhivkov, 'Generic Gâteaux differentiability of locally Lipschitz functions', in Proc. Conf. Constructive Function Theory '81, pp. 590-594 (Sofia, 1983).

[14] N.V. Zhivkov, 'Generic Gâteaux differentiability of directionally differentiable mappings', Rev. Roumaine Math. Pures Appl. 32 (1987), 179-188.

\author{
University of Sofia \\ Department of Mathematics and Informatics \\ 5 "Anton Ivanov" Boul. \\ Sofia 1126 \\ Bulgaria
}

